\title{
On-board Signal Predistortion for Digital Transparent Satellites
}

\author{
Nicolò Mazzali, Bhavani Shankar Mysore R, and Björn Ottersten \\ Interdisciplinary Centre for Security, Reliability and Trust \\ University of Luxembourg, Luxembourg \\ e-mails: \{nicolo.mazzali, bhavani.shankar, bjorn.ottersten\}@uni.lu
}

\begin{abstract}
On-board processing (OBP), a paradigm that allows for manipulation of the signal at the satellite transponder, is being embraced by a large section of the satellite community. Exploiting OBP can lead to efficient implementation, bandwidth saving, lower redundancy, and better performance. Enabling processing aboard a satellite necessitates re-assessing the implementation of a number of signal processing techniques which, so far, have been ground-centric. In this work, we investigate the possibility of implementing signal predistortion (SPD) aboard a satellite having digital transparent processor (DTP). Such satellites employ transponders that allow for the implementation of limited functionalities in the digital domain. On-board predistortion can then be performed on the digitized data and can provide better performance compared to on-ground techniques. However, the conversion to the digital domain performed by an analogto-digital converter (ADC) introduces different types of noise. Among these, the clock jitter requires the implementation of estimation and compensation algorithms. In this paper we propose a reduced-complexity on-board signal predistortion algorithm capable of post-compensating the jitter introduced by the ADC, and pre-compensating the distortion generated by the amplifier.

Index Terms - on-board processing, signal predistortion, clock jitter compensation, reduced-complexity algorithm
\end{abstract}

\section{INTRODUCTION}

Satellite communications, buoyed by new markets (e.g., maritime and aeronautical) and emerging applications (e.g., broadband internet, machine to machine, UHDTV/3DTV), are seeing a huge demand in terms of connectivity and throughput. Multi-beam architectures, along with novel signal processing and digital communication techniques like precoding for full frequency reuse [1], [2] and predistortion [3], have been proposed for efficient utilization of the existing spectrum. However, most of these techniques are implemented on-ground due to the use of transparent satellites where on-board signal processing is limited to channelization and amplification. While on-ground solutions provide for a low-complexity implementation, they are not necessarily efficient: for example, on-ground predistortion suffers from bandwidth restrictions on the feeder link as well as from the presence of input multiplexer (IMUX). Towards resolving these issues, there has been, of late, an investigation on the benefits of enabling on-board processing (OBP). Despite the idea not being recent [4], OBP has been neglected for many years by the research community mainly because of the several technological and feasibility issues raised by the satellite industry. OBP's core idea is to enable digital signal processing aboard the satellite in order to improve the performance of the system. Even though most of the currently employed civilian communication satellites are completely transparent, recent advances in device technology [5] have resulted in several satellites being provided with some on-board processing capability (e.g., SES-14 satellite).

There are two distinct types of transponders allowing OBP: those with digital transparent processing (DTP), and the fully regenerative (FR) ones. The payload of the FR transponders includes functionalities such as encoding/decoding, making the satellite deployment futuristic. On the other hand, the DTP transponder is an intermediate solution: no data-level functionality is available, but some digital operations can still be performed on the received digitized signal. For example, DTP can be used to implement efficient routing [6], or to increase the spectral efficiency of the feeder link [7].

Unfortunately, higher costs and complex payloads are not the only drawbacks of OBP. Along with the transition to the digital domain, new noise sources appear. The analogto-digital converter (ADC) introduces three main types of noise: quantization noise $(\mathrm{QN})$, clock jitter, and thermal noise [8]. QN can be mitigated by using a sigma-delta ADC [9] so that the residual noise can be modeled jointly with the thermal noise as zero-mean complex additive white Gaussian noise (AWGN). On the other hand, the clock jitter cannot be easily removed since its power spectral density (PSD) occupies the signal band [9]. Clock jitter is caused by phase noise affecting the clock oscillator of the ADC and generates a nonuniform sampling of the input signal. This mismatch leads to a degradation of the signal-to-noise ratio (SNR) inside the transponder [10]. In recent years, different approaches have been proposed so as to cope with its effects [11]-[13], but all of the proposed techniques suffer from a high complexity.

In order to asses the advantages of OBP, this paper focuses on DTP payloads and considers implementing on-board predistortion. The motivations for the choice of predistortion are twofold: (i) There is an increasing push towards countering the nonlinear impairments introduced by the on-board high power amplifier (HPA) to achieve higher power and spectral efficiencies [14]. (ii) Predistortion can be implemented by using the digital samples provided by the DTP.

Several on-ground techniques, like data predistortion (DPD) and signal predistortion (SPD), have been proposed to mitigate the channel nonlinear response. DPD processes constellation symbols [3], while SPD acts on the waveform [15], [16]. 
While each technique has its own pros and cons, DPD cannot be implemented with DTP-based payloads. Hence the focus of the paper is on the efficient on-board implementation of SPD. All the SPD techniques cause a spectral regrowth in the signal PSD that can be a serious problem in satellite communications, where transmitted signals have to satisfy tight emission masks. Nevertheless, OBP opens new ways to the SPD design. Indeed, while on-ground SPD has to deal with the stringent requirements of the emission masks, these constraints are weak or absent when implemented on-board. Moreover, an attractive way to cope with the high computational complexity of the SPD algorithms is resorting to a look-up table (LUT) approach for its digital implementation. Indeed, the LUT does not require the realization of nonlinear operations in hardware and can be fully implemented on a digital processor.

The contribution of this paper is a framework for the design of on-board SPD where the on-board impairments are taken into account. In particular, we design an SPD algorithm based on the statistical knowledge of the clock jitter. The proposed SPD post-compensates on average for the jitter and precompensates for the nonlinear distortion caused by the HPA. A LUT-based implementation is envisaged, and a novel iterative semi-analytical approach for identifying the predistorter parameters is derived. We will highlight the effects of on-board impairments and the gains provided by the proposed solution over a traditional on-ground approach.

\section{SCEnARIO And System Model}

We consider a typical Ka-band broadcast application from a geostationary satellite. The uplink signal from a gateway can either correspond to a single carrier or multiplexed carriers. In the latter case, we assume the carriers to correspond to different services. Finally, we assume a receiver capable of decoding only a single carrier. For the sake of simplicity, we neglect the interactions with the adjacent transponders. Moreover, since the focus of this paper is on on-board predistortion, we assume an ideal feeder link and an AWGN user link.

\section{A. DTP-based Payload}

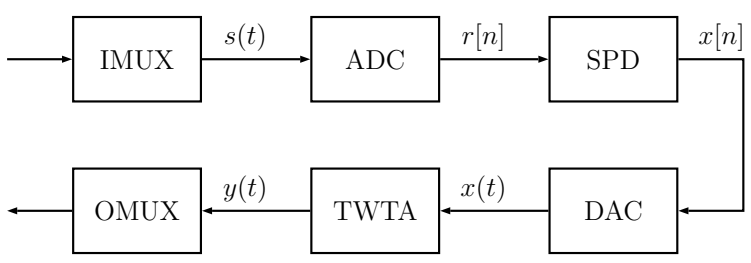

Fig. 1. Block scheme of the considered transponder with DTP.

We consider the payload architecture illustrated in Fig. 1, which depicts a DTP-based transponder comprising an IMUX filter, an ADC, a predistorter, a digital-to-analog converter (DAC), a traveling-wave tube amplifier (TWTA), and an output multiplexing (OMUX) filter. The IMUX filter can be either analog (as in transparent transponders) or digital. For the sake of simplicity, we have assumed it analog.
Let us denote by $s(t)$ the analog signal at the output of the IMUX filter. An ideal ADC would perform the digitization giving as output the sampled signal $s[n]=s\left(n T_{s}\right)$, where $T_{s}$ is the sampling interval ${ }^{1}$. In order to model the clock jitter, some approximations have to be introduced, and it can be shown that they hold under mild assumptions [13]:

1) the jitter sampled process $e[n]$ is a real $^{2}$ zero-mean colored Gaussian process with standard deviation $\sigma_{e}=$ $\eta T_{s}$, where $\eta \in[0,1]$

2) the jitter is small in comparison to $T_{s}$, i.e., $\eta \ll 1$;

3) variations in the jitter are much slower than the signal.

Residual QN and thermal noise are modeled as the sampled version of a single complex white Gaussian process $w(t)$ with mean zero and variance $\sigma^{2}$ per component. Under these assumptions, the input of the SPD can be described as

$$
r[n] \cong s[n]+e[n] \dot{s}[n]+w[n]
$$

where $\dot{s}[n]$ is the sampled first-order derivative of $s(t)$ [13]. The on-board SPD is then included, whose details are presented in Sections II-B and III. Subsequently, the output of the SPD is then converted into the analog domain by a DAC, assumed to be ideal. In other words, $x(t)$ is assumed to be the faithful reconstruction of the digital signal $x[n]$.

\section{B. Amplifier and Predistorter Modeling}

The input-output characteristics of the amplifier is a nonlinear non-invertible memoryless function, typically given as tabulated AM/AM and AM/PM characteristics. This function can be approximated by a memoryless third-order Volterra series expansion as

$$
y(t) \cong \alpha x(t)+\beta|x(t)|^{2} x(t)
$$

where the coefficients $\alpha$ and $\beta$ are assumed to be complex and have to be optimized offline to emulate the channel characteristics, as shown in Section III.

As SPD model we choose a memoryless third-order polynomial function, namely

$$
x[n]=\gamma r[n]+\delta|r[n]|^{2} r[n]
$$

since it provides for a satisfactory trade-off between performance and complexity [17]. Since we are focusing on the performance aboard the satellite and not at the user terminal, we will neglect the effects of the OMUX filter, assuming that they can be taken into account by the user terminal.

Two well-known methods to estimate the SPD parameters exist. Most of the predistortion algorithms in the literature resort to the indirect method, i.e., they approximately postinvert the channel and use the estimated post-inverse as predistorter [15]. On the other hand, the direct method, based

\footnotetext{
${ }^{1}$ We denote with squared brackets the sampled version of analog signals. Vectors are in bold lower case, and matrices in bold upper case. Symbols $\|$.$\| ,$ ${ }^{T},{ }^{H}, .^{*}$, and $\Re[$.$] denote the Euclidean norm, the transpose, the conjugate$ transpose, the conjugate, and the real part operators, respectively.

${ }^{2}$ It is worth noting that $e[n]$ is real-valued because it is approximately a scaled version of the difference between the ideally perfect phase and the noisy actual phase of the clock oscillator [13].
} 
on the pre-inversion on the channel, has been less investigated [16]. The SPD techniques based on the indirect method are affected by noisy measurements and by the assumption that a pre-compensation based on the post-inverse would give good performance results. In fact, nonlinear blocks do not commute and the chain performance does depend on the order of the nonlinear operations [16]. In Section III we will describe a novel iterative semi-analytical procedure to optimize the values of the SPD parameters. This procedure is based on the direct method and exploits the statistics of the jitter affecting the input of the SPD, as shown in Fig. 1 and modeled in (1).

\section{Predistorter Optimization}

The optimization of the predistorter coefficients is carried out in different stages, as described in the following.

\section{A. Parameter Estimation}

Assuming $\tilde{y}(t)$ to be the output of the TWTA computed by using the AM/AM and AM/PM characteristics, the optimal Volterra series coefficients $\alpha$ and $\beta$ can be jointly estimated by minimizing

$$
\alpha, \beta=\underset{\mathbb{C}^{2}}{\operatorname{argmin}} E_{x}\left\{\|\tilde{y}(t)-y(t)\|^{2}\right\}
$$

where $E_{x}\{$.$\} denotes the expectation with respect to the signal$ $x(t)$. This is a least squares minimization problem where the error function is linear in the coefficients $\alpha$ and $\beta$.

The SPD parameters, $\gamma$ and $\delta$, can be found by resorting to a similar minimization problem, i.e.,

$$
\gamma, \delta=\underset{\mathbb{C}^{2}}{\operatorname{argmin}} E\left\{\|y(t)-s(t)\|^{2}\right\}
$$

where $E\{$.$\} denotes the expectation with respect to the signal$ $s(t)$, the jitter $e[n]$, and the thermal noise $w[n]$. However, now the error function is nonlinear in the coefficients $\gamma$ and $\delta$ because of (2), and the method used to estimate the Volterra coefficients of the TWTA cannot be used any longer. Therefore, the minimization has to be done numerically. Unfortunately, finding a solution for (4) is a formidable task. Hence, we transform the minimization problem in (4) into an equivalent constraint-satisfaction problem that can be solved by exploiting the Divide \& Concur (D\&C) algorithm [18].

\section{B. Divide \& Concur Algorithm}

The $\mathrm{D} \& \mathrm{C}$ is an iterative heuristic algorithm widely used to solve constraint-satisfaction problems in many different fields [18]. D\&C introduces replicas of each variable, one for each constraint it is involved in. Different replicas of the same variable eventually have to equal each other, but temporarily they are allowed to be unequal while satisfying different constraints. In other words, temporary solutions are found for each constraint separately (possibly in parallel), and then melded into the final solution. For a more detailed description of the algorithm, its interpretation as a messagepassing algorithm, and some examples, the reader can refer to [18] and references therein.

The algorithm can be summarized in the following steps:
1) Divide Projection (DP): for each constraint, it moves the current replicas of the involved variables to the nearest values (in the Euclidean sense) that satisfy the constraint.

2) Concur Projection $(\mathrm{CP})$ : for each variable, it averages all its replica values.

The easiest way to combine the two steps is through alternating projections. By denoting as $\mathbf{c}_{k}$ the set of replicas at the $k$-th iteration, the resulting updating rule is

$$
\mathbf{c}_{k+1}=P_{C}\left(P_{D}\left(\mathbf{c}_{k}\right)\right)
$$

where $P_{D}($.$) denotes the DP and P_{C}($.$) the \mathrm{CP}$ as defined in [18]. Unfortunately, with this schedule the algorithm easily gets stuck in a trap (i.e., a loop where the replicas first satisfy the DP but not the CP, then they satisfy the CP but not the DP, and then go right back to the previous values satisfying the DP but not the CP). A way to escape from traps is the socalled Difference-Map (DM) dynamics [18], which modifies the update rule as

$$
\mathbf{c}_{k+1}=P_{C}\left(\mathbf{c}_{k}+2\left[P_{D}\left(\mathbf{c}_{k}\right)-\mathbf{c}_{k}\right]\right)-\left[P_{D}\left(\mathbf{c}_{k}\right)-\mathbf{c}_{k}\right]
$$

where the order of the projections (first DP and then CP) and the factor 2 are heuristic. In the following, we will adopt the update rule (5) and specialize it to the problem given by (4).

\section{Constraint-Satisfaction Formulation}

In order to convert the minimization problem in (4) into a constraint-satisfaction problem, we reformulate it by exploiting the first- and second-order necessary conditions for local minima. We then use these conditions as constraints in the D\&C algorithm. However, these conditions do not guarantee the determination of the global minimum and the solution provided by the D\&C algorithm is sensitive to the initialization. Therefore, a multi-start approach with random starting points is mandatory. For simplicity, the following analysis is carried out in the digital domain, by neglecting the ideal DAC and performing the optimization using the samples $y[n]$ instead of $y(t)$ as output of the TWTA.

First, we introduce the coefficient vector $\mathbf{c}=[\gamma, \delta]^{T}$ and the objective function

$$
f\left(\mathbf{c}, \mathbf{c}^{*}\right)=E\left\{\|\mathbf{y}-\mathbf{s}\|^{2}\right\}
$$

where we have defined $\mathbf{y}=[y[0], \ldots, y[N-1]]^{T}$ (respectively, s) as the vector containing $N$ consecutive samples of $y(t)$ (respectively, $s(t)$ ). The necessary first- and second-order conditions for local minima are

$$
\left\{\begin{array}{l}
\nabla_{\mathbf{c}^{*}} f\left(\mathbf{c}, \mathbf{c}^{*}\right)=\mathbf{0} \\
\mathcal{H} f\left(\mathbf{c}, \mathbf{c}^{*}\right) \quad \succeq 0
\end{array}\right.
$$

where $\nabla_{\mathbf{c}^{*}} f\left(\mathbf{c}, \mathbf{c}^{*}\right)$ denotes the conjugated complex gradient of the objective function, and $\mathcal{H} f\left(\mathbf{c}, \mathbf{c}^{*}\right)$ is the Hessian matrix, which has to be positive semi-definite [19]. In order to be practically implemented in the $\mathrm{D} \& \mathrm{C}$ algorithm, these two conditions have to be transformed into a set of equivalent realvalued constraints. The stationarity condition can be equivalently represented by four real-valued equality constraints, i.e., 
by imposing the real and the imaginary part of each of the two components of the gradient to be equal to zero. Since the Hessian matrix is Hermitian, it is positive semi-definite if and only if all of its principal minors are non-negative. Since $\mathcal{H} f\left(\mathbf{c}, \mathbf{c}^{*}\right)$ is a 4-by-4 dimensional matrix, this translates into fifteen real-valued inequality constraints.

In order to derive handier expressions for (7), we introduce the vector $\mathbf{r}=[r[0], \ldots, r[N-1]]^{T}$. By replacing (1)-(3) into (4), we can rewrite the objective function as

$$
f\left(\mathbf{c}, \mathbf{c}^{*}\right)=E\left\{\left\|\boldsymbol{\Phi}^{(1)} \mathbf{c}+\boldsymbol{\Phi}^{(3)}\left(\mathbf{c} \otimes \mathbf{c} \otimes \mathbf{c}^{*}\right)-\mathbf{s}\right\|^{2}\right\}
$$

where $\otimes$ denotes the Kronecker product, and where we have introduced the matrices ${ }^{3} \boldsymbol{\Phi}^{(1)}=\alpha\left[\mathbf{r},|\mathbf{r}|^{2} \mathbf{r}\right]$ and $\boldsymbol{\Phi}^{(3)}=\beta\left[|\mathbf{r}|^{2}\right.$ $\left.\mathbf{r},|\mathbf{r}|^{4} \circ \mathbf{r},|\mathbf{r}|^{4} \circ \mathbf{r},|\mathbf{r}|^{6} \circ \mathbf{r},|\mathbf{r}|^{4} \circ \mathbf{r},|\mathbf{r}|^{6} \circ \mathbf{r},|\mathbf{r}|^{6} \circ \mathbf{r},|\mathbf{r}|^{8} \circ \mathbf{r}\right]$. Matrices $\left\{\boldsymbol{\Phi}^{(i)}\right\}$ collect the SPD input signal (1) and its higher-order components generated by the cascade of SPD and TWTA, scaled by the estimated TWTA coefficients. An optimization with higher accuracy can be performed by considering a fifthorder Volterra series expansion instead of (2), and increasing the nonlinearity order in (3). However, we limit our analysis to the third-order case as a proof of concept. By expanding the Euclidean norm and performing the expectation with respect to the jitter and the thermal noise, (8) can be written as

$$
\begin{aligned}
f\left(\mathbf{c}, \mathbf{c}^{*}\right) & =\mathbf{c}^{H} \mathbf{A}_{11} \mathbf{c}+\left(\mathbf{c} \otimes \mathbf{c} \otimes \mathbf{c}^{*}\right)^{H} \mathbf{A}_{33}\left(\mathbf{c} \otimes \mathbf{c} \otimes \mathbf{c}^{*}\right) \\
& +2 \Re\left[\mathbf{c}^{H} \mathbf{A}_{13}\left(\mathbf{c} \otimes \mathbf{c} \otimes \mathbf{c}^{*}\right)-\mathbf{B}_{1} \mathbf{c}\right. \\
& \left.-\mathbf{B}_{3}\left(\mathbf{c} \otimes \mathbf{c} \otimes \mathbf{c}^{*}\right)\right]+\mathbf{s}^{H} \mathbf{s}
\end{aligned}
$$

where $\mathbf{A}_{i j}=E\left\{\boldsymbol{\Phi}^{(i) H} \boldsymbol{\Phi}^{(j)}\right\}$ and $\mathbf{B}_{i}=E\left\{\mathbf{s}^{H} \boldsymbol{\Phi}^{(i)}\right\}$. Since $e[n]$ is a real Gaussian process, only its even moments are nonzero. This means that nearly half of the terms resulting from the inner products $\left\{\boldsymbol{\Phi}^{(i) H} \boldsymbol{\Phi}^{(j)}\right\}$ and $\left\{\mathbf{s}^{H} \boldsymbol{\Phi}^{(i)}\right\}$ will be equal to zero. Moreover, the inner products act as a time average over $s[n]$ and its derivative. Since $s(t)$ and $\dot{s}(t)$ are assumed to be zero-mean (which is reasonable for a pass-band signal), only the terms having the form $a|\mathbf{s}|^{2 \ell}|\dot{\mathbf{s}}|^{2 m}$ (where $a$ is a real number, $\ell$ and $m$ are non-negative integers) need to be evaluated. Indeed, all the other terms asymptotically (in $N$ ) converge to zero. Finally, the thermal noise $w[n]$ has typically a variance much smaller than the jitter's one. This scale difference allows us to neglect all the terms depending on $\sigma^{2}$. Therefore, after some long algebra and by taking into account only the relevant nonzero terms, it is possible to rewrite the local minima conditions in (7) as functions of $\mathbf{c}$, $\left\{\mathbf{A}_{i j}\right\}$, and $\left\{\mathbf{B}_{i}\right\}$. We then can derive their equivalent realvalued constraints, omitted here for lack of space, that will be used by the D\&C algorithm.

\section{Look-Up Table Implementation}

Finally, the SPD algorithm can be summarized as follows:

1) Optimization of the TWTA parameters.

\footnotetext{
${ }^{3}$ The symbol $\circ$ represents the Hadamard product, and with a slight abuse of notation we denote by $|\mathbf{a}|^{n}$ a vector having $\left|a_{k}\right|^{n}$ as $k$-th entry (e.g., $\left.|\mathbf{a}|^{2}=\mathbf{a} \circ \mathbf{a}^{*}\right)$
}

2) Optimization of the SPD parameters by using $D \& C$ with DM update. The constraints are the one derived by replacing (9) in (7), and the variables are $\gamma$ and $\delta$.

3) LUT implementation of (3) on a DTP.

Since the optimization is performed offline, the LUT cannot follow the variations of the amplifier characteristics. An adaptive on-board SPD will be envisaged in future works.

\section{NumericAl RESUlts AND Discussion}

We choose the uplink signal to be a 16-APSK modulated DVB-S2X single-carrier signal with a roll-off 0.1 and a symbol rate of 27.5 MBaud [20]. As IMUX and TWTA we choose the reference responses described in [21], and scale the IMUX so as to have an OMUX with $-3 \mathrm{~dB}$ bandwidth of $38 \mathrm{MHz}$. For the offline optimization of the predistorter, $N=10^{5}$ randomly generated symbols are considered, as well as an oversampling factor $N_{s}=10$. The SNR of $s[n]$ with respect to $w[n]$ is assumed to be $65 \mathrm{~dB}$, which is much lower than the SNR in state-of-the-art ADCs [8], while the SNR on the downlink is assumed to be $15 \mathrm{~dB}$ [20]. For the jitter generation, a phase noise bandwidth of $1 \mathrm{MHz}$ has been considered [13].

Since the D\&C algorithm is iterative, it becomes imperative to impose a termination criterion. Hence, at each iteration of the $\mathrm{D} \& \mathrm{C}$ algorithm, the resulting predistorter coefficients are employed to compute (6) by using Monte Carlo simulations. To have an accurate representation of the system, the actual $\mathrm{AM} / \mathrm{AM}$ and $\mathrm{AM} / \mathrm{PM}$ characteristics are used instead of (2). The algorithm is terminated when the improvement in (6) is below $0.1 \%$ in 20 consecutive iterations. Then, the value of (6) is stored and the final choice of the optimal parameters is done by selecting the ones showing the lowest value.

We test the performance of our algorithm in terms of signal-to-interference-and-noise ratio (SINR) defined as $\operatorname{SINR}(\mathrm{OBO})=P_{S}(\mathrm{OBO}) /\left(P_{I}(\mathrm{OBO})+P_{N}(\mathrm{OBO})\right)$, where the signal power $P_{S}$, the interference power $P_{I}$, and the thermal noise power $P_{N}$ (accounting for both the noise onboard and in the downlink) are all functions of the output backoff (OBO) and are computed at the input of the receiver. The interference accounted in the SINR stems from the nonlinear interaction (caused by both SPD and TWTA) among the signal, the jitter, and the thermal noise. We compute the $\mathrm{OBO}$ and the SINR for different values of input back-off (IBO), ranging from $0 \mathrm{~dB}$ (i.e., heavy saturation) to $21 \mathrm{~dB}$ (i.e., linear region), with step-size of $3 \mathrm{~dB}$.

We first compare the ideal performance of the proposed SPD (i.e., with no jitter aboard) with its on-ground counterpart obtained by using D\&C. The OMUX artifacts are not considered and both predistorters are assumed memoryless for fair comparison. Fig. 2 shows that on-board SPD outperforms its on-ground counterpart both in terms of SINR (providing a 0.4 $\mathrm{dB}$ gain at $\mathrm{OBO}=4 \mathrm{~dB}$ ) and in terms of $\mathrm{OBO}$ (providing a $2 \mathrm{~dB}$ gain at SINR $=9.5 \mathrm{~dB}$ ). The on-ground SPD has to compensate for the IMUX as well, but since the chosen SPD function is memoryless, it is not suitable to deal with the memory introduced by the filter. Moreover, the IMUX cuts off the tails of the signal PSD, therefore signals with a higher 


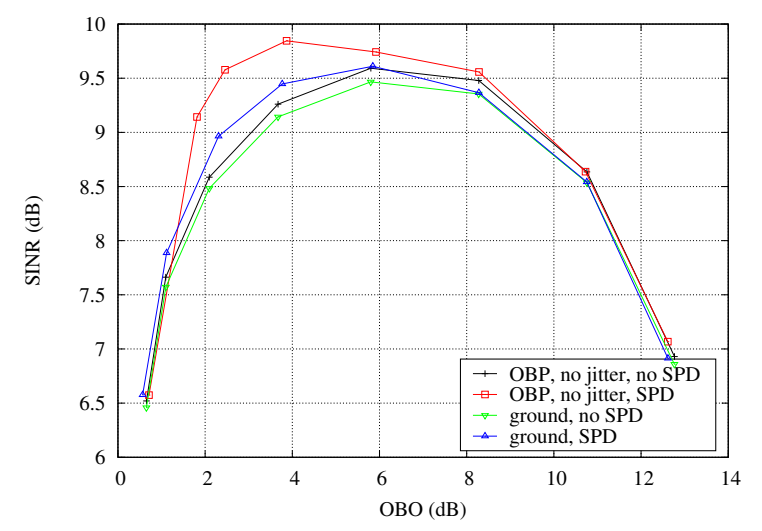

Fig. 2. SINR vs. OBO for the ideal OBP system with and without SPD, compared to the same on-ground algorithm.

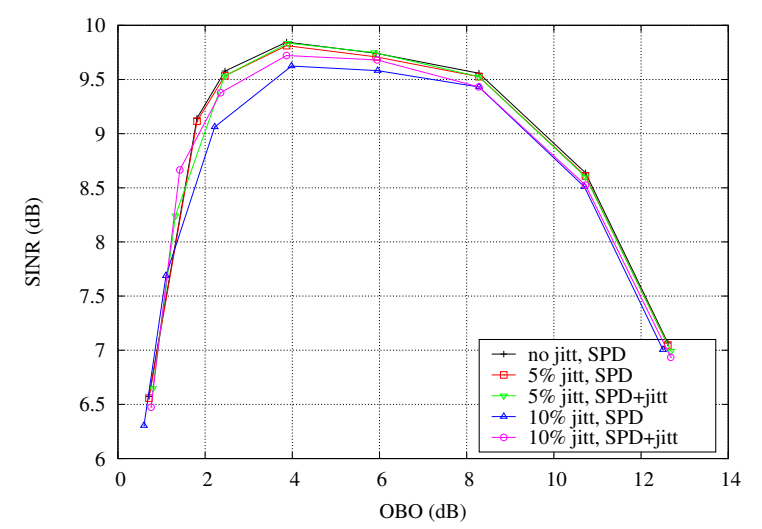

Fig. 3. SINR vs. OBO for the ideal OBP system, compared to the same system with SPD optimized taking into account the jitter or not, for jitter standard deviation $\sigma_{e}=0.05 T_{s}$ and $\sigma_{e}=0.1 T_{s}$.

baudrate would experience an additional degradation. Even with smaller SINR gains, the DVB-S2X standard allows to use different MODCODs with higher spectral efficiency [20], while the $\mathrm{OBO}$ gain is of interest to satellite operators because it translates into a more power-efficient amplification.

Fig. 3 shows that taking into account the jitter statistics during the optimization of the SPD coefficients is beneficial, even in the case of extremely high jitter (i.e., $\sigma_{e}=0.1 T_{s}$ ). Namely, by taking the jitter into account we can achieve an extra SINR gain of $0.1 \mathrm{~dB}$ (at $\mathrm{OBO}=4 \mathrm{~dB}$ ). For more realistic values of $\sigma_{e}$ (e.g., $\sigma_{e}=0.05 T_{s}$, which is still considered a very high value [13]), we can obtain nearly optimal performance. This allows us to discard any jitter compensation hardware from the satellite payload and resort to a cheaper and noisier ADC.

\section{CONCLUSiOnS}

In this paper we proposed a novel on-board SPD algorithm for satellites with DTP. The predistorter can efficiently precompensate for the distortion introduced by the nonlinear amplifier. Moreover, by taking into account the jitter statistics during the optimization process, it can also post-compensate for the jitter generated by the ADC. With the proposed SPD, a cheap and noisy ADC can be used and no dedicated hardware for de-jittering is required, allowing a reduction of the payload mass and the transponder power consumption. Finally, in order to further reduce the payload complexity, the proposed predistorter can be implemented directly in a DTP as a LUT.

\section{REFERENCES}

[1] D. Christopoulos, S. Chatzinotas, and B. Ottersten, "User scheduling for coordinated dual satellite systems with linear precoding," in Proc. IEEE Intern. Conf. Commun., June 2013, pp. 4498-4503.

[2] —, "Full frequency reuse multibeam SatComs: Frame based precoding and user scheduling," 2014. [Online]. Available: http://arxiv.org/abs/1406.7699

[3] R. Piazza, M. Bhavani Shankar, and B. Ottersten, "Data predistortion for multicarrier satellite channels based on direct learning," IEEE Trans. Signal Processing, vol. 62, no. 22, pp. 5868-5880, Nov 2014.

[4] L. Bella, "Multiservice on-board switching for mobile satellite communications," in Proc. IEEE Intern. Conf. Commun., Jun 1988, pp. 521525 , vol.1.

[5] Digital Transparent Processor, data sheet, Thales Alenia Space, Mar 2012.

[6] A. Le Pera, F. Fornì, M. Grossi, M. Lucente, V. Palma, T. Rossi, and M. Ruggieri, "Digital transparent processor for satellite telecommunication services," in IEEE Aerospace Conference, Mar 2007.

[7] E. Candreva, R. Suffritti, and M. Dervin, "Increasing the feeder link efficiency in broadband satellite systems," in Proc. Intern. Work. on Signal Processing for Space Commun., Sep 2014, pp. 300-305.

[8] K. Lauritzen, S. H. Talisa, and M. Peckerar, "Impact of decorrelation techniques on sampling noise in radio-frequency applications," IEEE Trans. Instr. Measur, vol. 59, no. 9, pp. 2272-2279, Sept 2010.

[9] B. Putra and G. Fettweis, "Clock jitter estimation and suppression in OFDM systems employing bandpass sigma-delta ADC," in Proc. IEEE Intern. Work. on Signal Processing Advances for Wireless Commun., Jun 2009, pp. 623-627.

[10] B. Razavi, RF Microelectronics, 2nd ed., Prentice-Hall, Ed., 2011.

[11] S. Hoyos, S. Pentakota, Z. Yu, E. Ghany, X. Chen, R. Saad, S. Palermo, and J. Silva-Martinez, "Clock-jitter-tolerant wideband receivers: An optimized multichannel filter-bank approach," IEEE Trans. Circuits and Systems I: Regular Papers, vol. 58, no. 2, pp. 253-263, Feb 2011.

[12] C. Anderson, S. Venkatesh, J. Ibrahim, R. Buehrer, and J. Reed, "Analysis and implementation of a time-interleaved ADC array for a software-defined UWB receiver," Proc. Vehicular Tech. Conf., vol. 58, no. 8, pp. 4046-4063, Oct 2009.

[13] Z. Towfic, S.-K. Ting, and A. Sayed, "Clock jitter compensation in highrate ADC circuits," IEEE Trans. Signal Processing, vol. 60, no. 11, pp. 5738-5753, Nov 2012.

[14] B. Beidas and R. Seshadri, "Analysis and compensation for nonlinear interference of two high-order modulation carriers over satellite link," IEEE Trans. Commun., vol. 58, no. 6, pp. 1824-1833, June 2010.

[15] L. Ding, G. Zhou, D. Morgan, Z. Ma, J. Kenney, J. Kim, and C. Giardina, "A robust digital baseband predistorter constructed using memory polynomials," IEEE Trans. Commun., vol. 52, no. 1, pp. 159-165, Jan 2004.

[16] D. Zhou and V. DeBrunner, "Novel adaptive nonlinear predistorters based on the direct learning algorithm," IEEE Trans. Signal Processing, vol. 55, no. 1, pp. 120-133, Jan 2007.

[17] E. Biglieri, S. Barberis, and M. Catena, "Analysis and compensation of nonlinearities in digital transmission systems," IEEE J. Select. Areas Commun., vol. 6, no. 1, pp. 42-51, Jan 1988.

[18] J. S. Yedidia, "Message-passing algorithms for inference and optimization," Journal of Statistical Physics, vol. 145, no. 4, pp. 860-890, 2011.

[19] A. Hjørungnes, Complex-Valued Matrix Derivatives With Applications in Signal Processing and Communications, C. U. Press, Ed., Mar 2011.

[20] ETSI EN 302 307-2 Digital Video Broadcasting (DVB), Second generation framing structure, channel coding and modulation systems for Broadcasting, Interactive Services, News Gathering and other broadband satellite applications, Part II: S2-Extensions (DVB-S2X), Available on ETSI web site (http://www.etsi.org).

[21] TM-S2 Channel Model Group, "DVB-SX channel models," Tech. Rep., 2012. 